\title{
The correlation between transverse momentum and multiplicity of charged particles in a two-component model
}

Evgeny Andronov*

Saint Petersburg State University

E-mail: evgeny.andronov1@gmail.com

Vladimir Vechernin ${ }^{\dagger}$

Saint Petersburg State University

E-mail: vechernin@gmail.com

\begin{abstract}
The long-range correlations between the transverse momenta (pT) and the multiplicities (n) of charged particles in two windows separated in rapidity are analyzed in the framework of the model with independent emitters (strings) of two types. Primary emitters are produced immediately in collision and secondary - after the interaction of some of the first ones. The mechanism of the emergence of secondary emitters is simulated by a simple toy model. The dependencies of the $n-n$ and pT-n correlations on the average number of primary emitters and on the parameter characterizing the strength of the interaction between them were studied. It was shown that with the increase of interaction parameter both the increase and the decrease of the $n$-n correlation strength are possible depending on the model parameters. The possibility of the model description of the experimentally observed negative pT-n correlations is discussed. The obtained analytical results were also tested by the Monte-Carlo simulations.
\end{abstract}

The XXI International Workshop High Energy Physics and Quantum Field Theory,

June 23-June 30, 2013

Saint Petersburg Area, Russia

\footnotetext{
* Speaker.

${ }^{\dagger}$ The work was supported by the RFFI grant 12-02-00356-a.
} 


\section{Introduction}

The study of the long-range particle correlations in the processes of the multiple production in high energy pp and AA collisions can provide us with information about behaviour of the produced high density matter. In this paper we study two types of the long-range correlations - the correlation between multiplicities of charged particles in two rapidity windows separated by some gap and the correlation between average transverse momentum and multiplicity in these windows. In order to characterize strength of the correlations the correlation parameters are usually introduced:

$$
b_{n-n}^{a b s}=\frac{\left\langle n_{F} n_{B}\right\rangle-\left\langle n_{F}\right\rangle\left\langle n_{B}\right\rangle}{\left\langle n_{F}{ }^{2}\right\rangle-\left\langle n_{F}\right\rangle^{2}}, \quad b_{p T-n}^{a b s}=\frac{\left\langle n_{F} p_{T B}\right\rangle-\left\langle n_{F}\right\rangle\left\langle p_{T B}\right\rangle}{\left\langle n_{F}{ }^{2}\right\rangle-\left\langle n_{F}\right\rangle^{2}},
$$

where the $n_{B}$ and $n_{F}$ are corresponding multiplicities in the "backward" and "forward" windows, $p_{t B}=\frac{1}{n_{B}} \sum_{i=1}^{n_{B}} p_{t B i}$ is the event mean transverse momentum for "backward" window. Symbol $\langle\ldots\rangle$ means an averaging over all events.

It is convenient to consider the correlations in the scaled variables (e.g. $n_{F} \rightarrow \frac{n_{B}}{\left\langle n_{B}\right\rangle}$ ) to get rid of the dependence on the width of the backward window. This rescaling gives:

$$
b_{n-n}^{r e l}=\frac{\left\langle n_{F}\right\rangle}{\left\langle n_{B}\right\rangle} b_{n-n}^{a b s}, \quad b_{p T-n}^{r e l}=\frac{\left\langle n_{F}\right\rangle}{\left\langle p_{T B}\right\rangle} b_{p T-n}^{a b s} .
$$

\section{General formalism}

The soft part of the particle production in high energy pp and AA collisions is often described in terms of color strings stretched between the partons of the projectile and the target [1-4]. The hadronization of these strings produces the observed hadrons. In this paper we generalize the model with independent identical emitters [5-7]. With the growing energy and the number of nucleons of colliding nuclei the number of emitters grows and it is possible that they will interact with each other changing resulting multiplicity and transverse momentum distributions. As a first step, we propose model with the emitters of two different types - primary emitters created at the beginning and secondary, produced after interaction of several primary emitters.

We assume that the probability $P\left(n_{B}, n_{F}\right)$ to observe the $n_{B}$ charged particles in the backward rapidity window and the $n_{F}$ charged particles in the forward rapidity window is given by the formula:

$$
P\left(n_{B}, n_{F}\right)=\sum_{C} \omega(C) P_{C}\left(n_{B}, n_{F}\right)
$$

where $C$ is the emitter configuration $\left(N_{1}, N_{2}\right), \omega(C)$ is the possibility to have this configuration and

$$
P_{C}\left(n_{B}, n_{F}\right)=\sum_{\left\{B_{i}^{(1)}, B_{j}^{(2)}\right\}} \delta_{n_{B}, \Sigma_{i=1}^{N_{1}} B_{i}^{(1)}+\sum_{j=1}^{N_{2}} B_{j}^{(2)}} \sum_{\left\{F_{i}^{(1)}, F_{j}^{(2)}\right\}} \delta_{n_{F}, \Sigma_{i=1}^{N_{1}} F_{i}^{(1)}+\sum_{j=1}^{N_{2}} F_{j}^{(2)}} \prod_{i=1}^{N_{1}} p_{1}\left(B_{i}^{(1)}, F_{i}^{(1)}\right) \prod_{j=1}^{N_{2}} p_{2}\left(B_{j}^{(2)}, F_{j}^{(2)}\right) .
$$

Here

$$
B_{i}^{(1)} \equiv n_{B}^{i(1)}, \quad F_{i}^{(1)} \equiv n_{F}^{i(1)}, \quad B_{j}^{(2)} \equiv n_{B}^{j(2)}, \quad F_{j}^{(2)} \equiv n_{F}^{j(2)}
$$


are the backward and forward multiplicities from the i-th primary emitter and the j-th secondary emitter. In the formula (2.2) $p_{1,2}\left(B_{i, j}^{(1,2)}, F_{i, j}^{(1,2)}\right)$ are the probabilities that the i-th primary $(\mathrm{j}$-th secondary) emitter produces the $B_{i}^{(1)}\left(B_{j}^{(2)}\right)$ charged particles in the backward window and the $F_{i}^{(1)}$ $\left(F_{j}^{(2)}\right)$ charged particles in the forward one. Delta symbols indicate that:

$$
n_{B}=B^{(1)}+B^{(2)}=\sum_{i=1}^{N_{1}} B_{i}^{(1)}+\sum_{j=1}^{N_{2}} B_{j}^{(2)} ; n_{F}=F^{(1)}+F^{(2)}=\sum_{i=1}^{N_{1}} F_{i}^{(1)}+\sum_{j=1}^{N_{2}} F_{j}^{(2)} .
$$

In case of the long-range correlations the model suppose that emitters produce particles independently in the forward and backward windows:

$$
p_{1}\left(B_{i}^{(1)}, F_{i}^{(1)}\right)=p_{B^{(1)}}\left(B_{i}^{(1)}\right) p_{F^{(1)}}\left(F_{i}^{(1)}\right) ; p_{2}\left(B_{j}^{(2)}, F_{j}^{(2)}\right)=p_{B^{(2)}}\left(B_{j}^{(2)}\right) p_{F^{(2)}}\left(F_{j}^{(2)}\right) .
$$

Then we have the factorization:

$$
P_{C}\left(n_{B}, n_{F}\right)=P_{C}\left(n_{B}\right) P_{C}\left(n_{F}\right)
$$

where

$$
\begin{aligned}
& P_{C}\left(n_{B}\right)=\sum_{\left\{B_{i}^{(1)}, B_{j}^{(2)}\right\}} \delta_{n_{B}} \sum_{i=1}^{N_{1}} B_{i}^{(1)}+\sum_{j=1}^{N_{2}} B_{j}^{(2)} \prod_{i=1}^{N_{1}} p_{B^{(1)}}\left(B_{i}^{(1)}\right) \prod_{j=1}^{N_{2}} p_{B^{(2)}}\left(B_{j}^{(2)}\right), \\
& P_{C}\left(n_{F}\right)=\sum_{\left\{F_{i}^{(1)}, F_{j}^{(2)}\right\}} \delta_{n_{F}} \sum_{i=1}^{N_{1}} F_{i}^{(1)}+\sum_{j=1}^{N_{2}} F_{j}^{(2)} \prod_{i=1}^{N_{1}} p_{F^{(1)}}\left(F_{i}^{(1)}\right) \prod_{j=1}^{N_{2}} p_{F^{(2)}}\left(F_{j}^{(2)}\right) .
\end{aligned}
$$

Now, we introduce the probability distribution for the event mean transverse momentum for the backward window. Let us denote by $k_{i x}^{(1)}\left(x=1, \ldots, B_{i}^{(1)}\right)$ the transverse momenta of particles produced by the $\mathrm{i}$-th primary emitter in the backward window and by $k_{j y}^{(2)}\left(y=1, \ldots, B_{j}^{(2)}\right)$ the transverse momenta of particles produced by the $\mathrm{j}$-th secondary emitter in the backward window. Then probability to have the event mean transverse momentum $p_{T B}$ in the backward window for given emitter configuration $\mathrm{C}$ and fixed numbers of particles $\left\{B_{i}^{(1)}, B_{j}^{(2)}\right\}$ produced by each emitter of the two types in the backward window is

$$
\begin{aligned}
& P_{\left\{B_{i}^{(1)}, B_{j}^{(2)}\right\}}^{C}\left(p_{T B}\right) \equiv \int \delta\left(p_{T B}-\left[\sum_{i=1}^{N_{1}} \sum_{x=1}^{B_{i}^{(1)}} k_{i x}^{(1)}+\sum_{j=1}^{N_{2}} \sum_{y=1}^{B_{j}^{(2)}} k_{j y}^{(2)}\right]\left[\sum_{i=1}^{N_{1}} B_{i 1}+\sum_{j=1}^{N_{2}} B_{i 2}\right]^{-1}\right) \times \\
& \times \prod_{i=1}^{N_{1} B_{i=1}^{(1)}} \rho_{1}\left(k_{i x}^{(1)}\right) d k_{i x}^{(1)} \prod_{j=1}^{N_{2}} \prod_{y=1}^{B_{j}^{(2)}} \rho_{2}\left(k_{j y}^{(2)}\right) d k_{j y}^{(2)}
\end{aligned}
$$

where $\rho_{1}(k)$ - the probability distribution of the transverse momentum for the primary emitter, $\rho_{2}(k)$ - from the secondary emitter. And again we have the factorization:

$$
P_{C}\left(p_{T B}, n_{B}, n_{F}\right)=P_{C}\left(p_{T B}, n_{B}\right) P_{C}\left(n_{F}\right)
$$


where

$$
P_{C}\left(p_{T B}, n_{B}\right)=\sum_{\left\{B_{i}^{(1)}, B_{j}^{(2)}\right\}} \delta_{n_{B}} \sum_{i=1}^{N_{1}} B_{i}^{(1)}+\sum_{j=1}^{N_{2}} B_{j}^{(2)} P_{\left\{B_{i}^{(1)}, B_{j}^{(2)}\right\}}^{C}\left(p_{T B}\right) \prod_{i=1}^{N_{1}} p_{B^{(1)}}\left(B_{i}^{(1)}\right) \prod_{j=1}^{N_{2}} p_{B^{(2)}}\left(B_{j}^{(2)}\right) .
$$

Using (2.1)-(2.10), we will derive expressions for the average values used in the definitions of the correlation coefficients (1.1), (1.2).

\section{3. n-n correlations}

Let us, at first, calculate $\left\langle n_{F}\right\rangle$ :

$$
\begin{aligned}
& \left\langle n_{F}\right\rangle \equiv \sum_{C} \omega(C)\left\langle n_{F}\right\rangle_{C}=\sum_{n_{F}} n_{F} \sum_{N_{1}, N_{2}} \omega\left(N_{1}, N_{2}\right) P_{N_{1}}\left(F^{(1)}\right) P_{N_{2}}\left(F^{(2)}\right)= \\
& =\sum_{N_{1}, N_{2}} \omega\left(N_{1}, N_{2}\right) \sum_{\left\{F_{i}^{(1)}, F_{j}^{(2)}\right\}}\left(\sum_{i=1}^{N_{1}} F_{i}^{(1)}+\sum_{j=1}^{N_{2}} F_{j}^{(2)}\right) \prod_{i=1}^{N_{1}} p_{F^{(1)}}\left(F_{i}^{(1)}\right) \prod_{j=1}^{N_{2}} p_{F^{(2)}}\left(F_{j}^{(2)}\right)= \\
& =\sum_{N_{1}, N_{2}} \omega\left(N_{1}, N_{2}\right)\left(N_{1} \bar{\mu}_{F^{(1)}}+N_{2} \bar{\mu}_{F^{(2)}}\right)=\bar{N}_{1} \bar{\mu}_{F^{(1)}}+\bar{N}_{2} \bar{\mu}_{F^{(2)}},
\end{aligned}
$$

where $\bar{\mu}_{F^{(1)}}, \bar{\mu}_{F^{(2)}}$ - the mean multiplicities from one primary/secondary emitter in the forward window, $\overline{N_{1}}, \overline{N_{2}}$ - the mean numbers of the primary/secondary emitters. Doing similar calculations for other averages we get the correlation coefficients:

$$
\begin{gathered}
b_{n-n}^{a b s}=\frac{D_{N_{1}} \bar{\mu}_{B^{(1)}} \bar{\mu}_{F^{(1)}}+\operatorname{cov}\left(N_{1}, N_{2}\right)\left(\bar{\mu}_{B^{(1)}} \bar{\mu}_{F^{(2)}}+\bar{\mu}_{B^{(2)}} \bar{\mu}_{F^{(1)}}\right)+D_{N_{2}} \bar{\mu}_{B^{(2)}} \bar{\mu}_{F^{(2)}}}{\overline{N_{1}} D_{\mu_{F^{(1)}}}+\bar{N}_{2} D_{\mu_{F^{(2)}}}+D_{N_{1}} \bar{\mu}_{F^{(1)}}{ }^{2}+D_{N_{2}} \bar{\mu}_{F^{(2)}}{ }^{2}+2 \operatorname{cov}\left(N_{1}, N_{2}\right) \bar{\mu}_{F^{(1)}} \bar{\mu}_{F^{(2)}}} \\
b_{n-n}^{r e l}=b_{n-n}^{a b s} \bar{N}_{{ }_{1}}{\overline{N_{1}}}_{F^{(1)}}+\bar{N}_{B^{(1)}}+\bar{\mu}_{F_{2}} \bar{\mu}_{B^{(2)}}
\end{gathered}
$$

where $D_{\mu_{F^{(1)}}}=\overline{\mu_{F^{(1)}}^{2}}-\bar{\mu}_{F^{(1)}}{ }^{2}, D_{\mu_{F^{(2)}}}=\overline{\mu_{F^{(2)}}^{2}}-\bar{\mu}_{F^{(2)}}{ }^{2}, \operatorname{cov}\left(N_{1}, N_{2}\right)=\overline{N_{1} N_{2}}-\overline{N_{1}} \overline{N_{2}}, D_{N_{1}}=\overline{N_{1}{ }^{2}}-$ ${\overline{N_{1}}}^{2}, D_{N_{2}}=\overline{N_{2}{ }^{2}}-{\overline{N_{2}}}^{2}$. If the properties of the primary and secondary emitters are the same, we come back to the result obtained in the framework of the model with identical emitters [7]:

$$
b_{n-n}^{a b s}=\frac{D_{N} \bar{\mu}_{B} \bar{\mu}_{F}}{\bar{N} D_{\mu_{F}}+\bar{\mu}_{F}^{2} D_{N}}, b^{r e l}{ }_{n-n}=\frac{D_{N} \bar{\mu}_{F}^{2}}{\bar{N} D_{\mu_{F}}+\bar{\mu}_{F}^{2} D_{N}} .
$$

To take into account the effect of interaction of the emitters the simple toy model was proposed. Let $N$ be the number of emitters produced immediately after collision. In papers $[2,8]$ it was implied that $N$ is even, because each pair of strings corresponds to one cut pomeron of the ReggeGribov approach. Then one can assemble $N$ emitters into $\frac{N}{2}$ pairs and introduce the probability $r$ of the pair transformation into one secondary emitter. As a result we will get a binomial distribution 


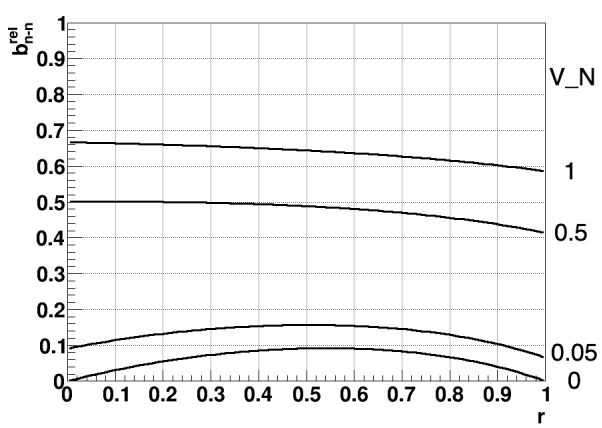

Figure 1: $b_{n-n}^{r e l}$ as a function of $r$ with $W_{\mu^{(1)}}=0.5$ and different values of $V_{N}=\{0 ; 0.05 ; 0.5 ; 1\}$

for the number of the secondary emitters with the fixed $N: P_{N}\left(N_{2}\right)=C_{\frac{N}{2}}^{N_{2}} \cdot r^{N_{2}} \cdot(1-r)^{\frac{N}{2}-N_{2}}$. Then $N_{1}=N-2 N_{2}$ and

$$
\sum_{N_{1}, N_{2}=0}^{\infty} \omega\left(N_{1}, N_{2}\right) \ldots=\sum_{N=0}^{\infty} \sum_{N_{2}=0}^{\frac{N}{2}} P_{N}\left(N_{2}\right) \cdot P(N) \ldots
$$

Also, it is possible to connect properties of the primary and secondary emitters using the String Fusion Model assumptions [5, 6]:

$$
\bar{\mu}_{F^{(2)}}=\sqrt{2} \bar{\mu}_{F^{(1)}} ; \bar{\mu}_{B^{(2)}}=\sqrt{2} \bar{\mu}_{B^{(1)}}, D_{\mu_{F^{(2)}}}=\sqrt{2} D_{\mu_{F^{(1)}}} .
$$

Then we get

$$
\begin{aligned}
& b_{n-n}^{r e l}=b_{n-n}^{a b s} \cdot \frac{\bar{N}_{1} \bar{\mu}_{F^{(1)}}+\bar{N}_{2} \bar{\mu}_{F^{(1)}} \sqrt{2}}{\bar{N}_{1} \bar{\mu}_{B^{(1)}}+\bar{N}_{2} \bar{\mu}_{B^{(1)}} \sqrt{2}}=b_{n-n}^{a b s} \cdot \frac{\bar{\mu}_{F^{(1)}}}{\bar{\mu}_{B^{(1)}}}= \\
& =\frac{r^{2}(\sqrt{2}-1)^{2}\left(\frac{V_{N}}{2}-1\right)+r(\sqrt{2}-1)\left(\sqrt{2}-1-\sqrt{2} V_{N}\right)+V_{N}}{r^{2}(\sqrt{2}-1)^{2}\left(\frac{V_{N}}{2}-1\right)+r(\sqrt{2}-1)\left(\sqrt{2}-1-\sqrt{2} V_{N}-\frac{W_{F^{(1)}}}{\sqrt{2}}\right)+V_{N}+W_{F^{(1)}}},
\end{aligned}
$$

where $W_{F^{(1)}} \equiv \frac{D_{F^{(1)}}}{\bar{\mu}_{F^{(1)}}^{2}}, V_{N} \equiv \frac{D_{N}}{\bar{N}}$.

In Fig. 1 the correlation coefficients for different values of parameters are presented as a function of interaction parameter $r$. One can see that $b_{n-n}^{r e l}$ can increase as well as decrease with the growth of the interaction strength in dependence of $V_{N}$. Another important feature is that with the increase of fluctuations in the number of initial emitters (i.e. increase of $V_{N}$ ) correlations also increase, but even in the absence of fluctuations in the number of initial emitters $\left(V_{N}=0\right)$ we still have correlations due to the interaction of emitters.

\section{4. pT-n correlations}

To get expression for the pT-n correlation coefficient we calculate additionally the following 
averages:

$$
\begin{aligned}
& \left\langle p_{T B}\right\rangle=\sum_{C} \omega(C)\left\langle p_{T B}\right\rangle_{C}=\sum_{B^{(1)}, B^{(2)}} \frac{\overline{k_{1}} B^{(1)}+\overline{k_{2}} B^{(2)}}{B^{(1)}+B^{(2)}} \sum_{N_{1}, N_{2}} \omega\left(N_{1}, N_{2}\right) P_{N_{1}}\left(B^{(1)}\right) P_{N_{2}}\left(B^{(2)}\right), \\
& \left\langle p_{T B} n_{F}\right\rangle=\sum_{C} \omega(C)\left\langle p_{T B}\right\rangle_{C}\left\langle n_{F}\right\rangle_{C}= \\
& =\sum_{B^{(1)}, B^{(2)}} \frac{\overline{k_{1}} B^{(1)}+\overline{k_{2}} B^{(2)}}{B^{(1)}+B^{(2)}} \sum_{N_{1}, N_{2}} \omega\left(N_{1}, N_{2}\right)\left(N_{1} \bar{\mu}_{F^{(1)}}+N_{2} \bar{\mu}_{F^{(2)}}\right) P_{N_{1}}\left(B^{(1)}\right) P_{N_{2}}\left(B^{(2)}\right),
\end{aligned}
$$

where $\overline{k_{1}}$ and $\overline{k_{2}}$ are the mean transverse momenta of charged particles in the backward window from one primary/secondary emitter.

It is not possible to perform these calculations explicitly, but one can replace denominators in (4.1), (4.2) with average value $B_{1}+B_{2} \approx \bar{N}_{1} \bar{\mu}_{B^{(1)}}+\bar{N}_{2} \bar{\mu}_{B^{(2)}}$. Then under assumption $\overline{k_{2}}=2^{\frac{1}{4}} \bar{k}_{1}[5,6]$ we get:

$$
\begin{aligned}
& b_{p_{t}-n}^{r e l}=b_{p_{t}-n}^{a b s} \cdot \frac{\left\langle n_{F}\right\rangle}{\left\langle p_{t B}\right\rangle}=b_{p_{t}-n}^{a b s} \cdot \frac{\bar{\mu}_{F^{(1)}} \bar{N} \cdot\left(1-r+\frac{r}{\sqrt{2}}\right)}{\overline{k_{1}} \cdot \frac{1-r+\frac{r}{2^{\frac{1}{4}}}}{1-r+\frac{r}{\sqrt{2}}}}= \\
& =\frac{\left(1-r+\frac{r}{\sqrt{2}}\right)^{2}}{1-r+\frac{r}{2^{\frac{1}{4}}}} \cdot \frac{\bar{N} \cdot\left(1-r+\frac{r}{2^{\frac{1}{4}}}-\left(1-r+\frac{r}{\sqrt{2}}\right) \sum_{N, N_{2}} P(N) C_{\frac{N}{2}}^{N_{2}} \cdot r^{N_{2}} \cdot(1-r)^{\frac{N}{2}-N_{2}} \cdot \frac{N-2 N_{2}+2^{\frac{3}{4}} N_{2}}{N-2 N_{2}+\sqrt{2} N_{2}}\right)}{r^{2}(\sqrt{2}-1)^{2}\left(\frac{V_{N}}{2}-1\right)+r(\sqrt{2}-1)\left(\sqrt{2}-1-\sqrt{2} V_{N}-\frac{W_{F_{F}(1)}}{\sqrt{2}}\right)+V_{N}+W_{\mu_{F}(1)}} .
\end{aligned}
$$

In (4.3) we still have the sum over $N$ and $N_{2}$. The calculation of this sum was performed numerically for the poissonian distribution $P(N)$ with $\bar{N}=50$ and for the fixed number of primary emitters $N=50$. Results of this calculation are presented in Fig. 2. One can see that in the both cases only negative pT-n correlations take place in the framework of this simple model. With the event growth of the portion of the secondary emitters multiplicity decreases while transverse momentum increases, leading to the negative pT-n correlation. For the configuration with only secondary emitters (at $r=1$ ) the correlations vanish due to the absence of fluctuations in the type of emitter. Note that negative pT-n correlations have already been observed in experiment [9] along with positive. We see that we need further modification of the model to describe this behaviour.

\section{Results and discussion}

In the framework of the model with independent emitters of two types the explicit formula for $b_{n-n}^{r e l}$ was obtained and the numerical calculations for $b_{p T-n}^{r e l}$ were performed. It was shown that with the inclusion of the interaction by the simple toy model n-n correlations can increase as well as decrease. At present only negative pT-n correlations were obtained in this framework.

One of the possible extensions of the model is the replacement of the constant interaction parameter $r$ with the function $r(N)$. It seems reasonable to suppose that with the growth of the number of emitters in transverse plane the probability to interact should also grow. 


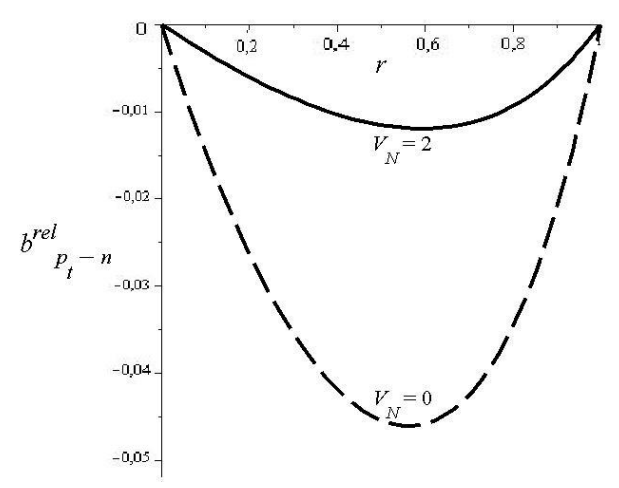

Figure 2: $b_{p T-n}^{r e l}$ as a function of $r$ with $W_{\mu^{(1)}}=0.5, \bar{N}=50$ and different values of $V_{N}=\{0 ; 2\}$

Note that in the last case ( $r$ as a function of $N$ ) one can not perform the analytical calculations of the correlation coefficients till the end, so the Monte Carlo simulations algorithm was developed and tested for constant $r$, giving values of correlation coefficients consistent with analytical calculations. This algorithm should be applied for $r(N)$, it should be checked if positive values of pT-n correlations are possible in this framework.

\section{References}

[1] A. Capella and A. Krzywicki, Phys. Rev. D 18 (1978) 4120.

[2] A. Capella, U.P. Sukhatme, C.-I. Tan and J. Tran Thanh Van, Phys. Lett. B 81 (1979) 68; Phys. Rep. 236 (1994) 225.

[3] A.B. Kaidalov, Phys. Lett. B 116, 459 (1982).

[4] A.B. Kaidalov, K.A. Ter-Martirosyan, Phys. Lett. B 117, 247 (1982).

[5] M.A. Braun, C. Pajares, V.V. Vechernin, Phys. Lett. B 493, 54 (2000) [hep-ph / 0007241 v1].

[6] M.A. Braun, R.S. Kolevatov, C. Pajares, V.V. Vechernin, Eur. Phys. J. C 32, 535 (2004) [hep-ph/0307056v1].

[7] V.V. Vechernin, Long-Range Rapidity Correlations in the Model with Independent Emitters, in proceedings of The XX International Baldin Seminar on High Energy Physics Problems, vol.2, JINR, Dubna (2011) 10 [1012.0214v1 [hep-ph] ].

[8] J. Dias de Deus, R. Ugoccioni, A. Rodrigues, Eur. Phys. J. C 16, 537 (2000) [hep-ph/9907352v1].

[9] NA49 Collaboration, G.A. Feofilov, R.S. Kondratiev, P.A. Naumenko, V.V. Vechernin, Long-Range Correlations in PbPb collisions at $158 \mathrm{AGeV}$, in proceedings of The XVII International Baldin Seminar on High Energy Physics Problems, vol.1, JINR, Dubna (2005) 222. 